\title{
Assessment of stem cell factor expression and its c-KIT receptor in patients with vitiligo
}

\author{
Dorota Wilamowska-Kokoszko', Karolina Łukasik², Jacek C. Szepietowski³ ${ }^{1}$ Dariusz J. Skarżynski², \\ Agnieszka Owczarczyk-Saczonek ${ }^{1}$, Justyna Hlebowicz-Skrodzka ${ }^{4}$, Waldemar J. Placek ${ }^{1}$
}

${ }^{1}$ Department of Dermatology, Sexually Transmitted Diseases and Clinical Immunology, University of Warmia and Mazury, Olsztyn, Poland

${ }^{2}$ Department of Reproductive Immunology and Pathology, Institute of Animal Reproduction and Food Research of the Polish Academy of Sciences, Olsztyn, Poland

${ }^{3}$ Department of Dermatology, Venereology and Allergology, Wroclaw Medical University, Wroclaw, Poland

${ }^{4}$ Department of Infectious Diseases and Hepatology, Medical University of Bialystok, Bialystok, Poland

Adv Dermatol Allergol 2022; XXXIX (4): 762-767

DOI: https://doi.org/10.5114/ada.2021.110266

\begin{abstract}
Introduction: Melanocytes show antigen expressions characteristic for the immune response effector cells, and the immune reactions in the skin, especially those with inflammation background, significantly affect the function of melanocytes. Among the cytokines produced by keratinocytes, the stem cell factor (SCF) plays a leading role in stimulating melanogenesis.

Aim: To compare the expression level of stem cell factor (mSCF, pSCF) and the c-Kit receptor in the centre of the vitiligo patch and in the area of healthy skin adjacent to the vitiligo patch.

Material and methods: The research material consisted of skin samples from a vitiligo lesion and from non-lesional skin adjacent to the vitiligo patch. Real Time PCR analysis (Applied Biosystems 7900HT) was performed to determine the expression level of the studied genes.

Results: The studies showed a statistically significant increase in the amount of mSCF within the vitiligo patch compared to both healthy skin of patients with vitiligo and controls. In patients with vitiligo, c-Kit receptor expression was significantly decreased in the area of the lesional skin compared to the healthy skin of the same patient and the skin of the control group.

Conclusions: The membrane-bound form of the SCF is overexpressed within the vitiligo skin, which may indicate the participation of $\mathrm{mSCF}$ in the stimulation of melanogenesis in response to melanocyte damage. Decreased expression of C-Kit receptor by melanocytes in the vitiligo patch disrupts the ligand-receptor interaction and may therefore be related to melanocytes dysfunction and/or loss.
\end{abstract}

Key words: vitiligo, stem cell factor, c-Kit receptor.

\section{Introduction}

Vitiligo is a hypopigmentary disorder and alteration in the expression level of several fibroblast-specific factors has been reported in the lesional skin of vitiligo patients. Melanocytes show antigen expression characteristic for immune response effector cells, and skin immune reactions especially those with inflammation, significantly affect the function of melanocytes, which can be seen as discoloration or depigmentation [1, 2]. The microenvironment of the epidermis plays an important role in melanocyte proliferation and melanogenesis. Keratino- cytes and melanocytes form a functional and anatomical connection called an epidermal melanin unit. Cytokines, produced by keratinocytes and fibroblasts, play a key role in its proper functioning $[3,4]$. The low levels of stem cell factor (SCF) and increased expression of tumour necrosis factor- $\alpha$ (TNF- $\alpha$ ) and interleukin-6 (IL-6), all of which negatively influence melanogenesis, have been reported when studying mRNA levels. It is especially important, considering that melanocytes express its receptor ckit and that SCF promotes melanocyte survival both in the initial migration and in the dermis [5]. Membrane

Address for correspondence: Dorota Wilamowska-Kokoszko MD, PhD, Department of Dermatology, Sexually Transmitted Diseases and Clinical Immunology, University of Warmia and Mazury, Al. Wojska Polskiego 30, 10-959 Olsztyn, Poland, phone: +48 896786654 , e-mail: dorotawilamowskakokoszko@gmail.com

Received: 7.02.2021, accepted: 25.06.2021. 
bound-type SCF (mSCF) is responsible for the interaction between keratinocytes and melanocytes and influences the survival and differentiation of melanocytes in the skin. The soluble form of SCF (SSCF) is involved in transferring melanoblasts from the neural crest cells to the skin and hair. It also regulates the interaction between melanocytes and fibroblasts $[6,7]$. The disturbance of cytokine homeostasis within the epidermal melanin unit leads to the intensification of melanocyte apoptosis [3]. UV irradiation directly induces the secretion of soluble SCF, which increases skin pigmentation. The soluble form SCF might be produced by the cleavage of surface bound SCF by several metalloproteases and might be released to the lumen of the vessels as well as secreted into extracellular spaces [8].

\section{Aim}

The aim of the study was to compare the expression level of the SCF (mSCF, SSCF) and the c-Kit receptor in the centre of the vitiligo patch and in the healthy skin adjacent to a vitiligo patch.

\section{Material and methods}

A group of 36 patients with vitiligo, including 26 women and 10 men, aged from 23 to 65 years, hospitalized in the Department of Dermatology, Sexually Transmitted Diseases and Clinical Immunology of the Municipal Hospital in Olsztyn or treated at the Dermatology Outpatient Clinic, were studied. The mean age of patients with vitiligo was 47.44 years, the mean age of women in this group was 49.84 years, and of men 41.2 years. Based on the Vitiligo Global Issues Consensus Conference - 2012, the study enrolled patients with non-segmental vitiligo. Patients with acral vitiligo were not included in the study group due to ineffectiveness of treatment, including PUVA-therapy, as proven in the literature.

The control group consisted of 40 healthy volunteers, including 28 women and 12 men, aged 25 to 62 years. The mean age of the control group is 47.61 years; the mean age of women -48.82 years, and men -44.89 years. The exclusion criterion in the control group was the presence of autoimmune or any other chronic diseases, any medications taken, any health complains, any abnormalities in laboratory tests (complete blood count; thyroid function tests: TSH, fT4, fT3, thyroid antibodies; C-reactive protein - according to the reference values of the laboratory of the Municipal Polyclinic Hospital in Olsztyn). Skin biopsy was collected using $5 \mathrm{~mm}$ biopsy punch after signing the informed consent, from the skin of the buttocks.

All patients and volunteers gave written consent to participation in the examination. The study was conducted in accordance with the recommendations of the Declaration of Helsinki and was approved by the Local
Bioethics Committee at the University of the Warmia and Mazury (decision no. 2/2015 of 14 January 2015). Written informed consent was obtained from all patients when they were enrolled.

Skin biopsy of both vitiligo patch and the healthy skin adjacent to the vitiligo patch was performed simultaneously in the same patients using $5 \mathrm{~mm}$ biopsy punch and then were used in the research. Samples of healthy skin were taken at least $5 \mathrm{~cm}$ away from vitiligo patch. The material was collected after signing the informed consent, from the skin not exposed to UV radiation. The tissues were protected in liquid nitrogen immediately after collecting the samples.

Real Time PCR (Applied Biosystems 7900HT) analyses were carried out at the Department of Reproductive Immunology and Pathology of the Institute of Animal Reproduction and Food Research of the Polish Academy of Sciences in Olsztyn. The head of the Department of Reproductive Immunology and Pathology in Olsztyn is prof. Dariusz Jan Skarżyński.

Skin samples were taken from the storage in tubes kept at $-80^{\circ} \mathrm{C}$ and homogenized using a ceramic mortar in liquid nitrogen to a form of fine tissue powder. Subsequently, samples were moved into a $1.5 \mathrm{ml}$ Eppendorf tube with $400 \mu \mathrm{l}$ of phenozol, stored for $30 \mathrm{~min}$, vortexed for $1 \mathrm{~min}$, and centrifuged. According to the manufacturer's protocol, total RNA from endometrial samples was isolated with the Total RNA Mini Plus kit (A\&A Biotechnology, Gdynia, Poland, \#036-100). The quantification of RNA was performed using the Nanodrop system (ND 200C; Fisher Scientific, Hampton, PA, USA) and its quality was assessed by visualization of 28S and $18 \mathrm{~S}$ rRNA bands after electrophoresis through a $1.5 \%$ agarose gel and red staining (41,003; Biotium, Hayward, CA, USA). The same amount (1000 ng) of RNA from each sample was reverse transcribed using Maxima First Strand cDNA Synthesis Kit (Thermo Fisher, Waltham, MA, USA, \#K1641). Reverse transcription was done according to the manufacturer's protocol. cDNA was frozen at $-20^{\circ} \mathrm{C}$ until further analysis. All the reactions for target and reference genes were performed in duplicate, on a 96-well plate (4306737; Applied Biosystems) and run in a ABI Prism 7900 sequence detection system (Applied Biosystems, Life Technologies, Foster City, CA, USA) with Maxima SYBR Green/ROX qPCR Master Mix (2X) (Thermo Scientific, Waltham, MA, USA, \#K0222). The samples' preparation was done according to the manufacturer's protocol using 15 ng cDNA per well in a total of $10 \mu \mathrm{l}$ of the reaction mix. The housekeeping gene was chosen according to the literature. Relative mRNA data were quantified using the quantitative PCR miner algorithm. Briefly, the average of the cyclic threshold (Cq) and the primer efficiency level (E) for each sample were related using the following equation: [1/(1pE) $\mathrm{Cq}$. Afterwards, the expression levels of the target genes were normalized against the $\beta$-actin - reference gene. 
Dorota Wilamowska-Kokoszko, Karolina Łukasik, Jacek C. Szepietowski, Dariusz J. Skarżynski, Agnieszka Owczarczyk-Saczonek, Justyna Hlebowicz-Skrodzka, Waldemar J. Placek

Table 1. Expression level of the membrane-bound form of stem cell factor (mSCF)

\begin{tabular}{|c|c|c|c|c|c|c|c|c|c|c|c|}
\hline $\mathrm{mSCF}$ & Group & $N$ & Mean & SD & Minimum & $\begin{array}{l}\text { Lower } \\
\text { quartile }\end{array}$ & Median & $\begin{array}{l}\text { Upper } \\
\text { quartile }\end{array}$ & Maximum & $P$-value* & $P$-value ${ }^{\star \star}$ \\
\hline \multirow[t]{2}{*}{ bz } & Patients & 36 & 0.0023 & 0.0009 & 0.0006 & 0.0017 & 0.0024 & 0.0032 & 0.0035 & 0.001 & 0.030 \\
\hline & Control & 40 & 0.0012 & 0.0005 & 0.0005 & 0.0009 & 0.0012 & 0.0018 & 0.0020 & & \\
\hline \multirow[t]{2}{*}{ bb } & Patients & 36 & 0.0042 & 0.0024 & 0.0012 & 0.0020 & 0.0038 & 0.0063 & 0.0090 & $<0.001$ & \\
\hline & Control & 40 & 0.0012 & 0.0005 & 0.0005 & 0.0009 & 0.0012 & 0.0018 & 0.0020 & & \\
\hline
\end{tabular}

bb-lesional skin biopsy sample, bz - healthy skin biopsy sample from a patient suffering from vitiligo. ${ }^{*}$ Comparison between the patient subgroup and the control subgroup (Mann-Whitney test). ${ }^{* *}$ Comparison between the skin sample of a vitiligo patch and the healthy skin of a person suffering from vitiligo, only a subgroup of patients (Wilcoxon's pairwise order test).

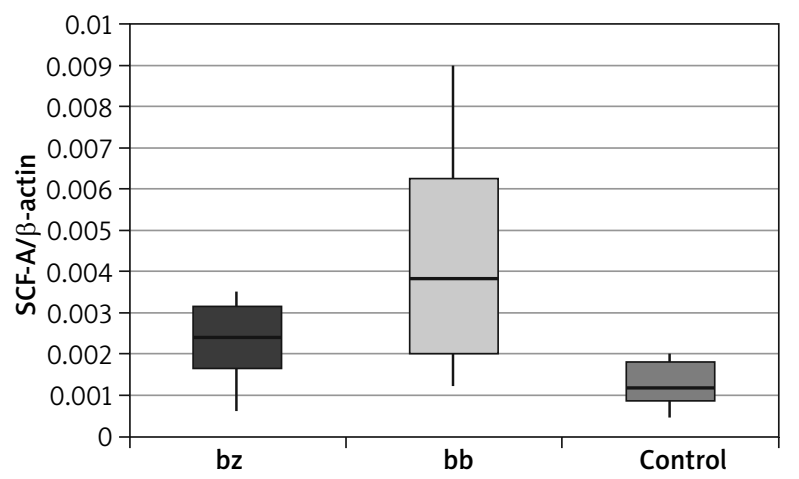

Figure 1. Expression level of the membrane-bound form of the stem cell factor

\section{Statistical analysis}

Statistical analysis was performed with the use of the IBM SPSS 20.0 package and the Microsoft Excel 2007. The Mann-Whitney test was used to evaluate the statistical significance of the differences in the results between the study group and the control group. The Wilcoxon pairwise test was used to compare the samples taken from healthy and lesional skin. For the proposed diagnostic tests, the measures of the diagnostic value of the test were calculated, such as sensitivity, specificity, positive predictive value (PPV) and negative predictive value (NPV), with the determination of their confidence intervals in relation to the final diagnosis. In all statistical tests, the level of statistical significance was set at $p<0.05$.

\section{Results}

In skin biopsy from vitiligo patches and healthy skin of vitiligo patients and those from the control group, the expression of the following genes was compared: the membrane-bound form of SCF (Table 1 and Figure 1), the soluble form of SCF (Table 2 and Figure 2) and the c-Kit receptor (Table 3 and Figure 3 ).

The calculation results showed that there was a statistically significant increase in the expression of the membrane-bound form of the SCF within the vitiligo patch compared to the healthy skin of patients suffering from vitiligo $(p=0.030)$ and to the control group ( $p<0.001)$. A statistically significant overexpression of mSCF was also demonstrated in healthy skin of people with vitiligo compared to the control group $(p=0.001)$.

The calculation results showed that there were no statistically significant differences between the expression of the soluble SCF within the study group (between healthy skin and vitiligo patches in patients with vitiligo). These differences were also not observed when comparing both the healthy skin and the lesional skin biopsy to the control group.

The calculation results showed that there was a statistically significant decrease in c-Kit receptor expression within the vitiligo patch compared to the healthy skin of vitiligo patients $(p=0.014)$ and in the vitiligo patch compared to the control group $(p=0.005)$. These differences were not observed when comparing the healthy skin of patients suffering from vitiligo and the control group.

Table 2. Expression level of the soluble stem cell factor (SSCF)

\begin{tabular}{|c|c|c|c|c|c|c|c|c|c|c|c|}
\hline sSCF & Group & $N$ & Mean & SD & Minimum & $\begin{array}{c}\text { Lower } \\
\text { quartile }\end{array}$ & Median & $\begin{array}{c}\text { Upper } \\
\text { quartile }\end{array}$ & Maximum & $P$-value* & $P$-value** \\
\hline \multirow[t]{2}{*}{ bz } & Patients & 36 & 0.0161 & 0.0093 & 0.0029 & 0.0090 & 0.0156 & 0.0203 & 0.0468 & 0.100 & 0.949 \\
\hline & Control & 40 & 0.0121 & 0.0093 & 0.0035 & 0.0048 & 0.0086 & 0.0174 & 0.0305 & & \\
\hline \multirow[t]{2}{*}{$\mathrm{bb}$} & Patients & 36 & 0.0155 & 0.0080 & 0.0057 & 0.0092 & 0.0123 & 0.0205 & 0.0385 & 0.088 & \\
\hline & Control & 40 & 0.0121 & 0.0093 & 0.0035 & 0.0048 & 0.0086 & 0.0174 & 0.0305 & & \\
\hline
\end{tabular}

bb-lesional skin biopsy sample, $b z$ - healthy skin biopsy sample from a patient suffering from vitiligo. ${ }^{*}$ Comparison between the patient subgroup and the control subgroup (Mann-Whitney test). ${ }^{* *}$ Comparison between the skin sample of a vitiligo patch and the healthy skin of a person suffering from vitiligo, only a subgroup of patients (Wilcoxon's pairwise order test). 
Table 3. C-Kit receptor expression level

\begin{tabular}{|c|c|c|c|c|c|c|c|c|c|c|c|}
\hline C-kit & Group & $N$ & Mean & SD & Minimum & $\begin{array}{l}\text { Lower } \\
\text { quartile }\end{array}$ & Median & $\begin{array}{c}\text { Upper } \\
\text { quartile }\end{array}$ & Maximum & $P$-value* & $P$-value ${ }^{\star \star}$ \\
\hline \multirow[t]{2}{*}{ bz } & Patients & 36 & 0.0247 & 0.0125 & 0.0103 & 0.0123 & 0.0207 & 0.0347 & 0.0546 & 0.076 & 0.014 \\
\hline & Control & 40 & 0.0357 & 0.0219 & 0.0183 & 0.0226 & 0.0295 & 0.0422 & 0.0927 & & \\
\hline \multirow[t]{2}{*}{ bb } & Patients & 36 & 0.0165 & 0.0119 & 0.0013 & 0.0038 & 0.0180 & 0.0271 & 0.0375 & 0.005 & \\
\hline & Control & 40 & 0.0357 & 0.0219 & 0.0183 & 0.0226 & 0.0295 & 0.0422 & 0.0927 & & \\
\hline
\end{tabular}

bb-lesional skin biopsy sample, bz - healthy skin biopsy sample from a patient suffering from vitiligo. *Comparison between the patient subgroup and the control subgroup (Mann-Whitney test). ${ }^{* *}$ Comparison between the skin sample of a vitiligo patch and the healthy skin of a person suffering from vitiligo, only a subgroup of patients (Wilcoxon's pairwise order test).



Figure 2. Expression level of the soluble stem cell growth factor (SSCF)

\section{Discussion}

The direct cause of the appearance of vitiligo patches is the selective destruction of melanocytes. Histopathological and immunopathological examinations of the skin of vitiligo patients showed the absence of melanocytes in the affected skin [9]. Despite numerous studies, the mechanism of the destruction of pigmented cells has not been fully understood yet [10]. It has been hypothesized that melanocytes together with keratinocytes form the so-called epidermal melanin unit [11]. Interactions between these two types of cells are important for the survival and differentiation of melanocytes due to cytokines released from keratinocytes, which act on melanocytes through specific receptors [12, 13]. The SCF/c-Kit pathway plays an important role in regulating the homeostasis of human melanocytes, increasing their number, size and density [14].

The available studies suggest that increasing the expression of melanogenic cytokines and chemokines, produced or released by keratinocytes, as well as increasing the number of receptors in response to various stimuli, stimulate melanogenesis. On the other hand, some authors believe that depigmentation is the result of point mutations both in specific receptors, e.g. c-Kit protein [15, 16] and in melanogenic cytokines, e.g. SCF [17-19]. Such defects usually result from disorders of melanoblast migration from the neural crests to the epidermis and hair follicles during foetal development. Due to the function of the SCF in the skin, it has been divided into two forms:

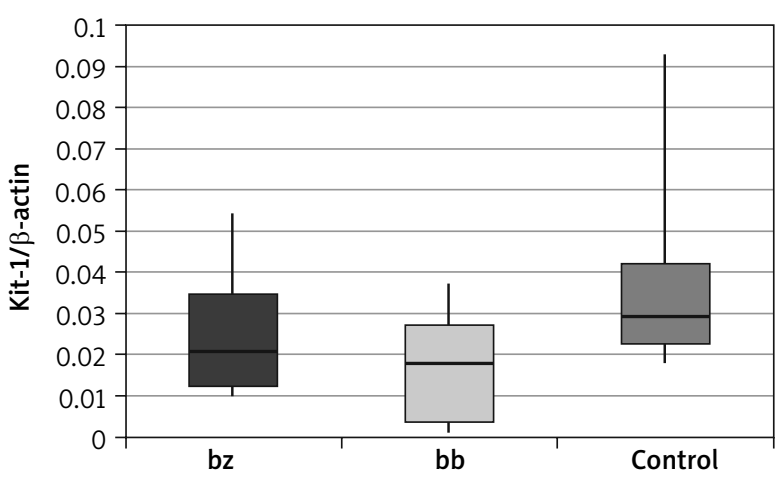

Figure 3. C-Kit receptor expression level

soluble SCF or SCF-1 and membrane bound SCF or SCF-2. The membrane-bound form (mSCF) influences the survival and differentiation of melanocytes in the skin, while the soluble form (SSCF) is involved in the transfer of melanoblasts from the neural crest cells to skin and hair [7]. Normal human keratinocytes contain mRNA of both SCF forms $[19,20]$. Lee et al. found that the number of normal human melanocytes in the culture increased in the presence of human keratinocytes and/or SCF, whereas the number decreased in a dose-dependent manner with the addition of anti-SCF antibodies [21].

Variable levels of melanogenic cytokines and chemokines are described in the available literature, confirming their participation in the pathogenesis of vitiligo. In case of SCF, the obtained data do not allow for firm conclusions, and in some cases the outcomes are mutually exclusive [9, 21, 22]. These differences may be caused by different research methodology and other criteria of selecting patients for the study group. Moreover, the reports cited above are connected with the level of SCF without division into the membrane-bound and soluble form.

Our research showed a statistically significant increase in the amount of mSCF within the vitiligo patch compared to both healthy skin of patients with vitiligo $(p=0.030)$ and control group $(p<0.001)$. The unexpected overexpression of mSCF indicates that the ability of keratinocytes to produce this melanogenic cytokine within the vitiligo lesion is not impaired. The obtained 
results may also be caused by the fact that the exclusion criterion was acral vitiligo. This assumption was based on a study [23] which compared the density of hair follicles, melanocytes, Langerhans cells (LC) and the expression of MHC class II, SCF and c-Kit receptor within the vitiligo area located in the repigmented regions (e.g. on the trunk) and acral vitiligo regions (the area of the lateral ankle and the palmar part of the wrist). They demonstrated a statistically significant decrease of all the above-mentioned parameters in acrally localized lesions compared to the central localization. This is probably caused by resistance to phototherapy in acral vitiligo. After a series of PUVA irradiations, the expression of the above parameters was re-measured, resulting in a reduction in SCF levels in both groups. The authors hypothesized that SCF depletion in vitiligo skin is due to binding of SCF to its c-Kit receptor in response to melanocyte damage and stimulation of melanogenesis through PUVA-therapy [23].

An interesting observation is the lack of statistically significant differences in the expression of the soluble form of SCF (SSCF) between the vitiligo skin and healthy skin of a person suffering from vitiligo $(p=0.949)$ and between the control group and the lesional skin ( $p=$ 0.088). Despite previously observed statistically significant differences between mSCF expression, this may indicate that SSCF is not involved in the keratinocyte response to melanocyte damage and stimulation of melanogenesis. The obtained results support the hypothesis that the soluble form of SCF is mainly involved in the transfer of melanoblasts from the neural crest cells to skin and hair during foetal life.

Analysing the c-Kit receptor expression measurements in vitiligo patients involved in the study, a statistically significant reduction in the amount of this protein was revealed in the lesional skin compared to the healthy skin of the same patient $(p=0.014)$. This difference was even more pronounced when the $\mathrm{c}$-Kit expression of the skin in the vitiligo patch was compared to the skin of the control group ( $p=0.005)$. An interesting observation, however, is the lack of statistically significant differences between the results obtained from the non-lesional skin of vitiligo patients compared to the control group $(p=0.076)$. Reduction of c-Kit protein expression by melanocytes within the vitiligo patch may be associated with dysfunction and/or loss of melanocytes. The c-Kit receptor plays another important role in the melanogenesis process, i.e. it activates MITF-M (melanocyte-specific microphthalmia-associated transcription factor) - a transcription factor associated with microphthalmia. Its expression occurs only in melanocytes and melanoma cells $[24,25]$ and it is considered to be the main regulator of melanogenesis at the transcriptional level. The MITF factor regulates the expression of genes specific for melanocytes, including tyrosinase, the TRP1 protein, which leads to melanocyte differentiation. Moreover, it activates the transcription of the melanocor- tin type 1 receptor (MC1R) for the $\alpha$-MSH hormone, which is responsible for the regulation of the melanogenesis process as well as the proliferation and formation of melanocyte projections $[25,26]$. The use of platelet-rich plasma (PRP), which contains several growth factors, has received attention as a treatment for stable vitiligo. More precisely, $\alpha$-melanocyte stimulating hormone ( $\alpha$-MSH), through its binding to MC1R, affects several melanocyte functions. This peptide plays a role in the regulation of melanogenesis, dendrite formation, and proliferation and differentiation, and it can also protect melanocytes from oxidative damage [27]. PRP is also known to boost hair growth. As the hair follicles act as reservoirs of melanocytes, a stimulating effect of PRP may be instrumental in repigmentation of leucotrichia [28].

\section{Conclusions}

The membrane-bound form of the SCF is overexpressed within the vitiligo skin, which may indicate the participation of mSCF in the stimulation of melanogenesis in response to melanocyte damage. Therefore, it seems that the ability of keratinocytes to produce this melanogenic cytokine within the vitiligo patch is not impaired. The soluble form of the SCF is not involved in the response of keratinocytes to melanocyte damage and stimulation of melanogenesis. It has also been shown that the expression of the c-Kit receptor by melanocytes is decreased within the vitiligo patch, which disturbs the ligand-receptor interaction and may be related to the dysfunction and/or loss of melanocytes.

\section{Conflict of interest}

The authors declare no conflict of interest.

\section{References}

1. Kapoor R, Dhatwalia SK, Kumar R, et al. Emerging role of dermal compartment in skin pigmentation: comprehensive review. J Eur Acad Dermatol Venereol 2020; 34: 2757-65.

2. Ruiz-Maldonado R, Orozco-Covarrubias ML. Postinflammatory hypopigmentation and hyperpigmentation. Semin $\mathrm{Cu}$ tan Med Surg 1997; 16: 36-43.

3. Placek W, Czajkowski R, Chabior A. Bielactwo nabyte. Dermatol Prakt 2009; 3: 9-18.

4. Mattei S, Colombo P, Melani C, et al. Expression of cytokine/ growth factors and their receptors in human melanoma and melanocytes. Int J Cancer 1994; 56: 853-7.

5. Seneschal J, Boniface K, D’Arino A, et al. An update on vitiligo pathogenesis. Pigment Cell Melanoma Res 2020, https:// doi.org/10.1111/pcmr.12949.

6. Imokawa G. Autocrine and paracrine regulation of melanocytes in human skin and in pigmentary disorders. Pigment Cell Res 2004, 17: 96-110.

7. Welker P, Schadendorf D, Artuc M, et al. Expression of SCF splice variants in human melanocytes and melanoma cell lines: potential prognostic implications. Br J Cancer 2000; 82: 1453-8. 
8. Kim M, Shibata T, Kwon S, et al. Ultraviolet-irradiated endothelial cells secrete stem cell factor and induce epidermal pigmentation. Sci Rep 2018; 8: 4235.

9. Moretti S, Spallanzani A, Amato L, et al. New insights into the pathogenesis of vitiligo: imbalance of epidermal cytokines at sites of lesions. Pigment Cell Res 2002; 15: 87-92.

10. Bystryn JC. Serum antibodies in vitiligo patients. Clin Dermatol 1989; 7: 136-45.

11. Jimbow K. Some aspects of melanin biology: 1950-1975. J Invest Dermatol 1976; 67: 72-8.

12. Gordon PR, Mansur CP, Gilchrest BA. Regulation of human melanocyte growth, dendricity, and melanization by keratinocyte derived factors. J Invest Dermatol 1989; 92: 565-72.

13. Kondo S. The roles of keratinocyte-derived cytokines in the epidermie and their possible responses to UVA irradiation. J Invset Dermatol 1999; 4: 177-83.

14. Grichnih JM, Burch JA, Burchette J, et al. The SCF/KIT pathway plays a critical role in the control of normal human melanocyte homeostasis. J Invest Dermatol 1998; 111: 233-8.

15. Giebel LB, Spritz RA. Mutation of the KIT (mast/stem-cell growth factor receptor) protooncogene in human piebaldism. Proc Natl Acad Sci USA 1991; 88: 8696-9.

16. Ezoe K, Holmes SA, Ho L, et al. Novel mutations and deletions of the KIT (steel factor receptor) gene in human piebaldism. Am J Hum Genet 1995; 56: 58-66.

17. Williams DE, Eisenman J, Baird A, et al. Identification of a ligand for the c-Kit proto-oncogene. Cell 1990; 63: 167-74.

18. Flanfan JG, Leder P. The kit ligand: a cell surface molecule altered in steel mutant fibroblasts. Cell 1990; 63: 185-94.

19. Zsebo KM, Williams DA, Geissler EN, et al. Stem cell factor is encoded at the SI locus of the mouse and is the ligand for the c-Kit tyrosine kinase receptor. Cell 1990; 63: 213-24.

20. Welker P, Grabbe J, Gibbs B, et al. Human mast cells produce and differentially express both soluble and membranebound stem cell factor. Scand J Immunol 1999; 49: 495-500.

21. Lee AY, Kim NH, Choi WI, et al. Less keratinocyte-derived factors related to more keratinocyte apoptosis in depigmented than normally pigmented suction-blistered epidermis may cause passive melanocyte death in vitiligo. I Invest Dermatol 2005; 124: 976-83.

22. Kitamura R, Tsukamoto K, Harada K, et al. Mechanisms underlying the dysfunction of melanocytes In vitiligo epidermis: role of SCF/KIT protein interactions and the downstream effector, MITF-M. J Pathol 2004; 202: 463-75.

23. Esmat SM, El-Tawdy AM, Hafez GA, et al. Acral lesions of vitiligo: why are they resistant to photochemotherapy? J Eur Acad Dermatol Venerol 2012; 26: 1097-104.

24. Das PK, van den Wijngaard RM, Wankowicz-Kalinska A, et al. A symbiotic concept of auto-immunity and tumor immunity: lessons from vitiligo. Trends Immunol 2001; 22: 130.

25. Drukała J, Bobis S, Żabińska-Płazak E, et al. Molekularne podłoże zaburzeń pigmentacji w chorobach skóry. Przegl Lek 2009; 66: 145-9.

26. Šamija I, Lukač J, Kusić Z. Microphthalmia-associated transcription factor (MITF) - from Waardenburg syndrome genetics to melanoma therapy. Acta Med Acad 2010; 39: 175-9.

27. Migayron L, Boniface K, Seneschal J. Vitiligo, From physiopathology to emerging treatments: a review. Dermatol Ther 2020; 10: 1185-98.

28. Garg S, Namrata Dosapaty N, Arora AK. Laser ablation of the recipient area with platelet-richplasma-enriched epidermal suspension transplant in vitiligo surgery: a pilot study. Dermatol Surg 2018; 45: 1. 